\title{
Conhecimento e prática em UTI Neonatais brasileiras: a perspectiva de seus gestores sobre a implementação de diretrizes clínicas
}

I Maria Auxiliadora Sousa Mendes Gomes,

2 Susana Maciel Wuillanume, ${ }^{3}$ Cynthia Magluta I

Resumo: A superação da lacuna entre o conhecimento estabelecido e sistematizado em diretrizes clínicas e a prática dos serviços de saúde é um desafio para a qualidade do cuidado. Diretrizes clínicas são ferramentas que sistematizam a evidência científica, auxiliando a identificar aquelas relevantes ao cuidado apropriado. Diversas estratégias para ampliar o uso de diretrizes clínicas foram identificadas na literatura: distribuição de material educacional; encontros para a capacitação; revisão das práticas; visita de especialistas; e lembretes. A análise de tais estratégias na perspectiva dos gestores de UTIN foi o objeto do estudo, desenvolvido em UTIN públicas através da aplicação de questionário semiestruturado com 53 chefias (médica e de enfermagem). Os entrevistados relataram a utilização concomitante de várias atividades/estratégias, concordando com posicionamentos da literatura. Cerca de $40 \%$ dos chefes consideraram que a distribuição de material escrito incentiva a adesão dos profissionais. A maioria dos gestores relatou que utiliza frequentemente as reuniōes para discussão de diretrizes clínicas, sendo esta uma atividade recomendada por mais de $95 \%$ deles. No contexto estudado, verificou-se que as estratégias para ampliar a disseminação do conhecimento dependem dos próprios profissionais e serviços, não havendo apoio institucional formal para seu financiamento, o que reduz a consistência de seu uso.

> Palavras-chave: Guias de Práticas Clínicas; implementação; neonatologia.

\author{
1 Doutora em Ciências pela \\ Pós-graduação de Saúde da \\ Mulher e da Criança. Núcleo de \\ Avaliação de Tecnologias em \\ Saúde do IFF, docente da Pós- \\ graduação de Saúde da Mulher \\ e da Criança do IFF. Endereço \\ eletrônico: maria@iff.fiocruz.br \\ ${ }^{2}$ Doutora em Ciências pela \\ Pós-graduação de Saúde da \\ Mulher e da Criança. Docente \\ do Departamento de Ensino \\ do IDD e da Pós-graduação de \\ Saúde da Mulher e da Criança \\ do IFF. Endereço eletrônico: \\ susanamw@iff.fiocruz.br \\ ${ }^{3}$ Doutora em Ciências pela \\ Pós-graduação de Saúde da \\ Mulher e da Criança. Unidade \\ de Pesquisa Clínica / Núcleo de \\ Avaliação de Tecnologias em \\ Saúde. Endereço eletrônico: \\ cynthiamagluta@globo.com
}




\section{Introdução}

O volume de informações científicas na área da saúde cresce de forma importante, desafiando os profissionais a reconhecer, dentre elas, quais são relevantes para o cuidado apropriado (CABANA et al., 1999).

As diretrizes clínicas (DC), bem construídas e baseadas em evidências científicas, têm sido consideradas ferramentas importantes na busca pela melhor qualidade da assistência (WOOLF et al., 2002) e pela redução das variações entre serviços e entre profissionais, uma vez que resumem e sistematizam as melhores evidências. As diretrizes podem ser ainda consideradas como um instrumento de capacitação profissional (GRECO; EISENBERG, 1993).

No Brasil, no contexto do cuidado neonatal, variações indesejáveis de práticas foram identificadas por vários pesquisadores: subutilização do corticóide antenatal (REDE, 2004), manejo inadequado da dor no bebê submetido a procedimentos dolorosos durante sua internação na UTIN (PRESTES et al., 2005), emprego tardio do surfactante e baixa utilização da ventilação gentil (MEDEIROS, 2006).

Diversos autores enfatizam a dificuldade da superação da lacuna entre o conhecimento produzido, e Grinshaw et al. (2008), em uma meta-análise sobre as diferentes estratégias de disseminação e implementação das diretrizes clínicas, identificaram efeitos variados (de pequeno a moderado) na adoção de novas diretrizes pelos serviços, sendo o maior efeito observado quando as estratégias são aplicadas em conjunto.

As estratégias identificadas abrangem: distribuição de material educacional; encontros para a capacitação, incluindo aqueles realizados no serviço e em congressos; a revisão das práticas com o retorno da informação à equipe assistencial; visita de um especialista ao local de prática dos profissionais para disseminar as diretrizes; lembretes para o processo assistencial, que podem ser dados oralmente, escritos ou utilizando sistemas informatizados; e, finalmente, intervençôes mediadas pelos pacientes, o que implica repassar informações à sociedade, possibilitando que ela, quando interaja com os profissionais, possa solicitar um cuidado específico.

$\mathrm{O}$ objetivo da pesquisa foi analisar as práticas referidas pelos gestores de Unidade de Terapia Intensiva Neonatal (UTIN) de maternidades públicas 
brasileiras sobre as atividades para disseminação e implementação de diretrizes clínicas em seus serviços.

\section{Metodologia}

Foi realizado um inquérito em uma amostra de conveniência de UTIN, composta por dois grupos de unidades, sendo o primeiro composto pelas unidades localizadas no município do Rio de Janeiro, aqui denominada Rede Rio de Janeiro (RRJ), e o segundo grupo, pelas unidades que compunham a Rede Brasileira de Pesquisas Neonatais (RBPN).

Participaram do inquérito oito das dez unidades públicas da RRJ, sendo seis da rede municipal e duas pertencentes a hospitais federais. Foram incluídas sete das oito unidades integrantes da RBPN, constituída por serviços de hospitais universitários. As perdas foram decorrentes da dificuldade de agenda da equipe, ou de reestruturação da equipe.

A maioria das UTIN foi classificada como IIIB (COMMITEE, 2004) por dispor de estrutura para cuidar de recém-nascidos de extremo baixo peso ( $\mathrm{PN}<$ $1000 \mathrm{~g}$ ) e por prover suporte de vida e acesso a procedimentos cirúrgicos menores. Duas unidades (25\%) da RRJ eram de complexidade IIIA, por não terem disponibilidade de cirurgia. Uma unidade da RBPN referiu cuidar de recémnascidos no pós-operatório de cirurgia cardíaca, sendo classificada como IIIC. A relação com hospital geral caracterizou a RBPN (86\%), sendo pouco frequente na RRJ (38\%).

No tocante à estrutura, a RBPN dispunha de $75 \%$ de suas UTIN com mais de 15 leitos, enquanto a RRJ tinha apenas 25\% de suas unidades com essa quantidade. A maioria das unidades (80\%) apresentou um volume assistencial grande, com mais de 2000 nascidos vivos por ano, sendo que $73 \%$ das UTIN assistiram mais de 3\% de RN com muito baixo peso.

As informações do estudo foram obtidas por meio de um questionário com questões estruturadas e semiestruturadas e de uma observação, realizada durante uma visita ao serviço, com duração trinta minutos.

O questionário foi respondido pelas equipes gestoras das UTIN, fazendo parte delas as chefias médicas e de enfermagem e os profissionais indicados por eles como seus substitutos e que tivessem um papel de liderança na equipe. 
Uma pergunta estruturada apresentava uma listagem com algumas estratégias para disseminação e implementação de DC identificadas na literatura (GRIMSHAW et al, 2004; TAMBLYN, 1993) e solicitava que o gestor indicasse seu posicionamento a respeito delas numa escala de 5 pontos, havendo possibilidade do entrevistado incluir novas alternativas.

Para cada atividade de implementação das DC, havia questionamentos sobre as quatro dimensōes identificadas na literatura (CABANA et al, 1999; GRIMSHAW et al, 2004), a saber: (I) a frequência de utilização da atividade para implementar as DC; (II) a estimativa do gasto dos diversos tipos de recursos, financeiros e materiais, e o tempo do gestor e dos profissionais; (III) a adesão dos profissionais às atividades; e (IV) a mudança de comportamento clínico dos profissionais, visando saber se os gestores perceberam a adoção das diretrizes propostas. Em seguida, foi solicitado ao gestor que selecionasse quais atividades recomendaria para outros serviços do SUS que desejassem implementar as DC.

Procurou-se, na visita à UTIN, identificar se existiam informações acessíveis para subsidiar o trabalho, tais como DC escritas, cartazes, fluxogramas, acesso à internet. Verificou-se, ainda, se havia um local para reunião próximo à UTIN.

O questionário foi validado por três médicos e três enfermeiros especialistas em neonatologia. O índice alpha Cronbach calculado para todos os itens em conjunto foi de 0,97 e, em relação a cada segmento do questionário, foi superior a 0,81, resultados superiores ao valor de 0,75 recomendado na literatura (WALLACE et al., 2003). O instrumento foi, ainda, testado com médico e enfermeiro com experiência de gestão de UTI Neonatal.

As entrevistas ocorreram em encontros de cerca de 40 minutos no período de julho a março de 2008 e foram gravadas em áudio. Uma equipe gestora da RBPN (um médico e uma enfermeira) optou por enviar suas respostas por correio eletrônico. Na sistematização das questôes semiestruturadas, utilizaramse, de forma complementar, as respostas escritas pelos chefes nos questionários, as anotações da pesquisadora no momento da entrevista e as transcrições das gravaçóes das entrevistas. $\mathrm{Na}$ análise, buscou-se integrar os achados obtidos através das perguntas estruturadas com aqueles obtidos com as questões semiestruturadas, visando ampliar a compreensão do aspecto tratado. Os dados foram sistematizados e quantificados. 
Os entrevistados foram descritos nas seguintes características: sexo; idade; tempo de prática de neonatologia; tempo (em anos) de ocupação do cargo de chefia; qualificação em neonatologia e em gestão. A qualificação em neonatologia para a chefia é uma exigência legal (BRASIL, 2000), e a capacitação para a gestão foi incluída tomando por referência o debate sobre a necessidade de qualificação dos gestores (MOTTA, 1991), considerando-se desejável que eles tenham algum tipo de qualificação específica para o exercício da gestão.

O projeto foi submetido e aprovado pelo Comitê de Ética em Pesquisa do Instituto Fernandes Figueira (CAAE 0014.1.008-08) e também obteve aprovação por quatro outros CEPS, pertencentes aos hospitais que assim a solicitaram (CAAE 0082.0.314.000-08 - SMS/RJ, CAAE 0082.0.318.008-08 - HGB, CAAE 0061.0.002.008-09 - PUC RGS, Protocolo do CEP/HSE 000.346). Em todas as entrevistas, foi obtido o consentimento informado.

\section{Resultados}

\section{Características dos entrevistados}

Foram entrevistados 53 chefes de UTIN, sendo 30 da RRJ e 23 da RBPN. A maioria tem mais de 40 anos de idade (74\%), mais de 10 anos de prática profissional em neonatologia (92\%) e ocupa o cargo de chefia há mais de 5 $\operatorname{anos}(51 \%)$.

A qualificação em neonatologia foi referida por $84 \%$ dos chefes, e a qualificação para gestão, seja através de cursos de especialização ou atualização, foi relatada por cerca de $40 \%$ dos gestores da RRJ.

\section{Estratégias para disseminação e implementação das DC}

Em todos os serviços, foi possível verificar a existência de um livro ou pasta com as diretrizes clínicas do serviço em local acessível para a equipe de rotina ou plantão. Em todos, havia um espaço para a reunião de equipe, embora ele também se destinasse a múltiplos usos. Somente numa unidade esse local não era próximo da UTIN.

$\mathrm{O}$ acesso à internet foi distinto entre as duas redes, ocorrendo somente em uma unidade da RRJ (13\%) enquanto seis (86\%) da RBPN dispunham de tal recurso, e também da listagem dos endereços eletrônicos dos profissionais. 
Os chefes entrevistados relataram a utilização concomitante de diversas atividades para a disseminação de DC, sendo que $60 \%$ dos gestores da RRJ e 91\% da RBPN utilizavam todas as atividades apresentadas no questionário, com diferença, porém, na intensidade de utilização de cada uma. Segundo afirmação de um deles, o fator crítico para essa disseminação é a comunicação, e todos meios deveriam ser tentados para possibilitar a compreensão das DC, evitando que os profissionais, quando questionados sobre sua conduta discordante das DC do serviço, utilizem como argumento não conhecê-las.

A divulgação de DC por meio de material escrito foi reportada de forma distinta entre as redes (tabela 1). Dentre os chefes da RRJ, a maioria (63\%) mencionou usar material impresso, a anotação no livro do serviço, e $61 \%$ dos gestores da RBPN relataram o envio de material por correio eletrônico.

Alguns dos entrevistados pertencentes ao grupo de chefes da RRJ (20\%) disseram que arcavam com os custos dessas atividades, utilizavam o computador de sua casa para fazer pesquisa na internet e pagavam a impressão e reprodução, o que limitava o uso dessa alternativa, uma vez que não consideravam adequado realizar tais gastos. A estratégia de um serviço da RBPN foi a produção de um livro próprio, que foi vendido aos profissionais e alunos. Cerca de $40 \%$ dos chefes de ambas as redes consideraram que a distribuição de material escrito incentiva a adesão dos profissionais.

A revisão de prontuários seguida por discussão com a equipe foi utilizada de forma frequente por 53\% dos gestores da RRJ e 35\% dos gestores da RBPN, que utilizam menos esse processo que não utilizam esse processo por possuírem bancos de dados. Mais da metade dos gestores da RRJ considerou que essa atividade tem boa adesão dos profissionais, e sua aplicação foi recomendada em outros serviços do SUS pela maioria dos gestores da RRJ (77\%) e da RBPN (61\%) (tabela 1 e gráficos 1 e 2). 
Tabela 1. Posicionamento dos gestores sobre as estratégias a eles apresentadas para implementar as DC, indicando frequencia de uso, intensidade de adesão e da mudança de comportamento dos profissionais e segundo a rede a que pertence, 2009

\begin{tabular}{|c|c|c|c|c|c|c|}
\hline \multirow{3}{*}{$\begin{array}{c}\text { Estratégias para implementar as } \\
\text { diretrizes clínicas apresentadas aos } \\
\text { gestores }\end{array}$} & \multicolumn{6}{|c|}{ Distribuição percentual dos gestores (\%) } \\
\hline & \multicolumn{2}{|c|}{$\begin{array}{l}\text { Frequência alta } \\
\text { de uso }\end{array}$} & \multicolumn{2}{|c|}{ Adesão Intensa } & \multicolumn{2}{|c|}{$\begin{array}{c}\text { Mudança } \\
\text { intensa de } \\
\text { comportamento }\end{array}$} \\
\hline & RRJ & RBPN & RRJ & RBPN & RRJ & RBPN \\
\hline \multicolumn{7}{|l|}{ Distribuição de material educacional } \\
\hline Anota no livro do serviço & 63 & 22 & 33 & 22 & 27 & 17 \\
\hline Distribui material escrito & 27 & 35 & 40 & 43 & 43 & 35 \\
\hline Envia mensagens eletrônicas & 7 & 61 & 7 & 39 & 7 & 39 \\
\hline \multicolumn{7}{|l|}{ Auditoria com retorno da informação } \\
\hline $\begin{array}{l}\text { Revisa prontuários e discute os } \\
\text { resultados }\end{array}$ & 53 & 35 & 50 & 35 & 60 & 39 \\
\hline \multicolumn{7}{|l|}{ Encontro para capacitação } \\
\hline \multicolumn{7}{|l|}{ No cotidiano do cuidado } \\
\hline Utiliza a passagem de plantão & 37 & 70 & 30 & 70 & 43 & 61 \\
\hline Utiliza a visita diária aos pacientes & 53 & 78 & 60 & 78 & 73 & 87 \\
\hline \multicolumn{7}{|l|}{ Reuniões agendadas } \\
\hline Utiliza reuniōes para discutir as DC & 57 & 83 & 33 & 65 & 43 & 61 \\
\hline Apresenta DC em reunião & 37 & 61 & 40 & 52 & 50 & 26 \\
\hline Promove aulas teóricas & 13 & 43 & 17 & 35 & 27 & 39 \\
\hline Discute casos clínicos & 27 & 48 & 20 & 43 & 47 & 57 \\
\hline \multicolumn{7}{|l|}{ Visita educacional de especialista } \\
\hline Convida um especilista & 7 & 17 & 37 & 52 & 47 & 48 \\
\hline \multicolumn{7}{|l|}{ Lembretes } \\
\hline Afixa cartazes & 43 & 43 & 53 & 39 & 57 & 35 \\
\hline $\begin{array}{l}\text { Desenvolve formulários para o } \\
\text { prontuário }\end{array}$ & 30 & 39 & 37 & 61 & 37 & 57 \\
\hline $\begin{array}{l}\text { Utiliza lembretes para orientar a } \\
\text { prática }\end{array}$ & 50 & 48 & 47 & 52 & 53 & 48 \\
\hline
\end{tabular}


Gráfico 1. Atividades para implementação de DC: uso e recomendação dos gestores da RRJ

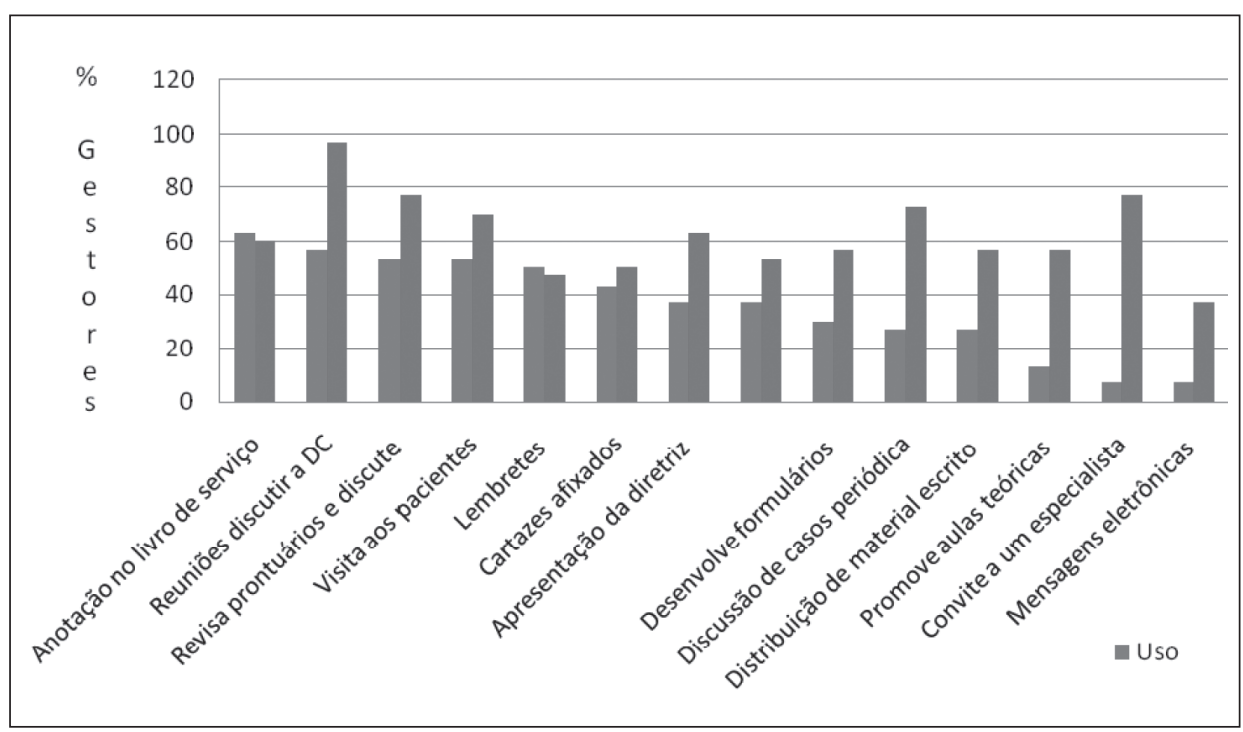

Gráfico 2. Atividades para implementação de DC: uso e recomendação dos gestores da RBPN

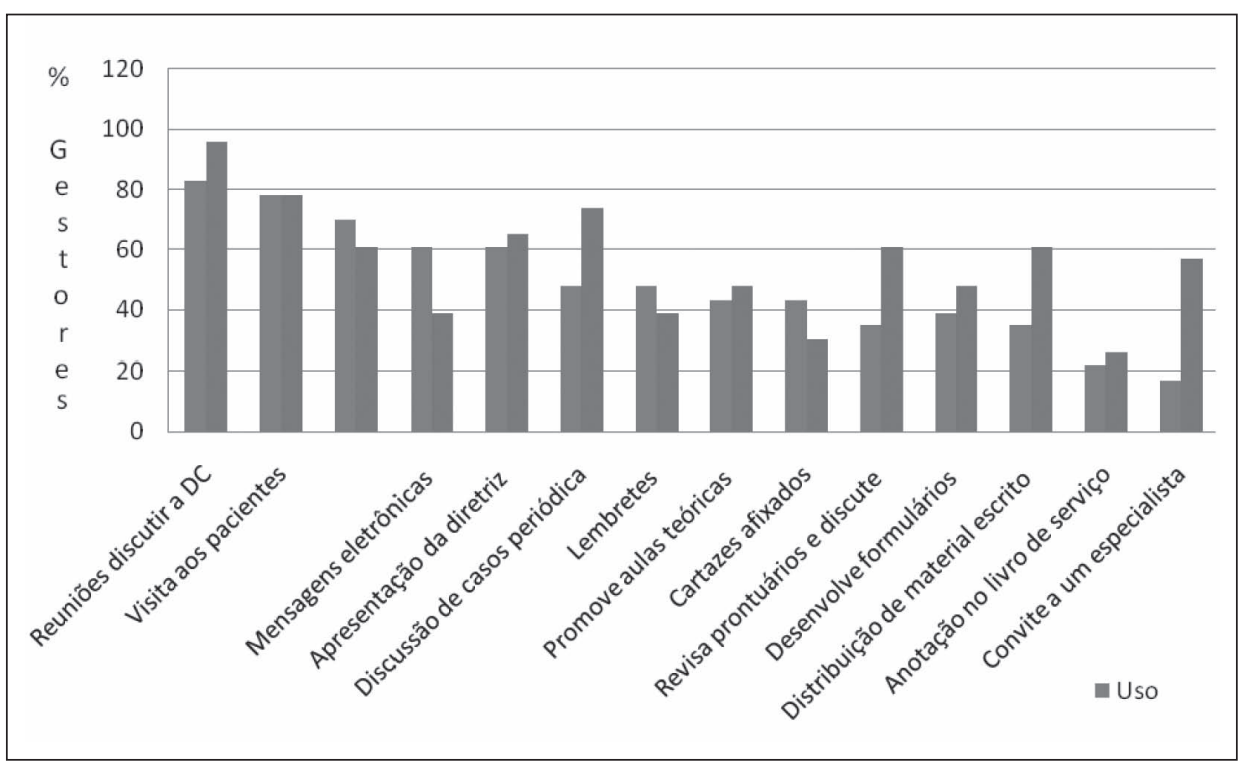


Seis atividades foram incluídas como atividades para capacitação. A maior parte dos gestores (57\% na RRJ e $83 \%$ na RBPN) relatou que utiliza frequentemente as reuniōes para discussão de diretrizes clínicas, sendo essa uma atividade recomendada por mais de $95 \%$ deles (tabela 1 e gráfico 1 e 2). A avaliação da adesão a essa atividade foi relatada de forma distinta pelos gestores: apenas 33\% dos gestores da RRJ percebem boa adesão dos profissionais, enquanto 65\% dos gestores da RBPN tiveram esse posicionamento.

Um gestor da RRJ ressaltou que os profissionais da rotina comparecem mais frequentemente às discussões por já estarem presentes no serviço, e que os plantonistas não se deslocam para essas atividades. Para dois gestores da RBPN, o comparecimento dos profissionais é garantido, como parte das atribuições dos docentes. Mais gestores da RBPN (61\%) do que do RRJ (43\%) consideraram que essa atividade tem boa capacidade de promover mudanças em suas práticas, sendo a mais recomendada por eles, mais de 95\% (Tabela 1 e Gráfico 1 e 2).

Dentre os encontros para capacitação, duas atividades de repasse de informação foram pesquisadas: a apresentação da diretriz em reunião e a aula teórica para os profissionais da equipe. Fazia-se a distinção entre a aplicação dessas atividades para os profissionais e para alunos, distinção reforçada especialmente para os gestores da RBPN, por serem serviços universitários e que têm essas atividades para os alunos. O relato de utilização dessas modalidades de informação foi distinto para os gestores da RBPN, sendo mais intensa a utilização da primeira atividade (61\%), se comparada à segunda (43\%).

Poucos gestores da RRJ relataram utilizar atividades de repasse de informações, sendo que 37\% fazem apresentações das DC e 13\% utilizam a aula teórica, justificando que a dificuldade de praticá-las é a disponibilidade de tempo, não apresentando argumentos contrários ao repasse de informação. A menor valorização dessas atividades de repasse de informação, se comparadas às atividades de discussão, pode ser inferida pelo menor percentual dos que as recomendam (RRJ - 48\% e RBPN - 65\%).

Também se procurou saber se a discussão das DC ocorria em duas atividades do cotidiano dos serviços: a passagem de plantão e a visita diária para acompanhamento dos pacientes. A primeira foi relatada por $70 \%$ dos gestores da RBPN e por metade dos gestores da RRJ. Alguns posicionamentos foram no 
sentido de valorizar esse momento do cotidiano do cuidado para reforçar aspectos das diretrizes, compreendendo que os plantonistas não comparecem fora de seu horário de trabalho para outras atividades. Ressaltaram que esse pode ser um momento importante para diálogo com a equipe, mas que depende da presença do chefe, e que isso não ocorre frequentemente, pois significaria um ajuste no cotidiano de trabalho para os gestores e a repetição da informação a cada plantão.

Alguns chefes de enfermagem (4) relataram que se organizam para ter a passagem do plantão como um momento privilegiado de contato com a equipe, possibilitando a analise dos pacientes com os prontuários em mãos. Uma das chefes indicou que não é a equipe de assistência que tem que vir ao encontro da de capacitação, mas que esta é que precisa estar à disposição daquela. No entanto, elas referem que são momentos de contato curto, devido ao cansaço da equipe que sai do plantão e porque as tarefas do dia devem começar a ser realizadas.

A visita para acompanhamento do paciente tem sido mais frequentada pelos profissionais da rotina do que pelos plantonistas, e utilizada de forma intensa pela maioria dos gestores (53\% na RRJ e 78\% na RBPN), com boa adesão dos profissionais ( $60 \%$ na RRJ e $78 \%$ na RBPN) e boa capacidade de contribuição para a mudança de prática clínica ( $73 \%$ na RRJ e $87 \%$ na RBPN), sendo também recomendada por $74 \%$ dos gestores, a segunda atividade mais recomendada na RBPN.

O convite a um especialista para falar para o grupo foi relatado por somente dois gestores da RRJ (7\%) e por quatro da RBPN (17\%). Esses últimos, por terem serviços localizados em hospitais universitários, relataram ter acesso aos especialistas do próprio hospital, ou que aproveitam a presença do especialista do país ou do exterior, convocado para outras atividades acadêmicas, para concretizar o convite, não tendo, em geral, despesas para as instituiçôes. Alguns entrevistados valorizaram o papel do especialista, reconhecendo que com seu expertise podem convencer os profissionais a utilizar as DC.

O último grupo de atividades incluídos na pesquisa foram os lembretes, ou seja, textos, bilhetes, cartazes e formulários que orientam os profissionais durante o cuidado. Os formulários são os menos referidos, com frequência em torno de $30 \%$. Os cartazes foram citados por cerca de $40 \%$ dos gestores. Em diversos serviços, durante a observação das instalaçōes da UTIN, foram identificadas tabelas e informações afixadas nos locais de prescrição e também cartazes 
lembrando as lavagens de mãos visando ao controle da infecção hospitalar, afixados próximo às pias - alguns deles já danificados pelo tempo de exposição.

Os lembretes foram mencionados indicados por cerca de 50\% dos gestores, sendo seu uso indicado para situações específicas, como na RBPN, onde foram utilizados para lembrar protocolos de pesquisa ou como alertas aos profissionais, especialmente aos plantonistas, quando se identifica conduta discordante daquela prevista. Entretanto, alguns gestores se posicionaram de forma contrária ao seu uso, argumentando que se poderia comprometer a privacidade do paciente e de sua família.

Os entrevistados recomendaram a utilização de diversas atividades em conjunto, divergindo um pouco sobre qual grupo de atividades seria escolhido. As mais recomendadas foram: discussão em reunião (96\%); visita para acompanhamento dos pacientes (74\%); discussões de casos (74\%); e a revisão de prontuários com discussão dos resultados (70\%) (gráficos 1 e 2).

\section{Discussão}

Os entrevistados são gestores de UTIN de hospitais de referência, com qualificação em neonatologia, vivenciando tanto a especialidade como a gestão por período significativo, qualificando-os como interlocutores importantes para a pesquisa. Os posicionamentos dos gestores da RRJ e da RBPN não se diferenciaram de forma importante em relação às atividades para implementação de diretrizes clínicas, sendo que as diferenças encontradas pareciam refletir os contextos institucionais distintos.

Apesar de considerar que as evidências científicas revisadas no período de 30 anos ainda não tenham a robustez necessária para subsidiar opções dos gestores e dos sistemas de saúde, a meta-análise desenvolvida por Grinshaw et al. (2004) indica que nem todas as estratégias têm a mesma possibilidade de influenciar o comportamento clínico dos profissionais ou de convencê-los a adotar uma nova conduta no cuidado dos pacientes.

Na bibliografia consultada sobre disseminação e implementação de DC, encontram-se frequentemente as seguintes afirmativas: o envio de material instrucional e as palestras têm sido estratégias mais empregadas, mas são consideradas pouco efetivas para a mudança de prática (GRINSHAW et al., 2004; BERO et al., 1988; VILLAR; CARROLI; GULMEZOGLU, 2001). O 
uso concomitante de diversas atividades é mais efetivo (SCOTT et al., 2004; CAMITINI, 2005). O papel do chefe ou dos líderes clínicos do serviço é fundamental (DIJKSTRA et al., 2006) e a participação dos profissionais na discussão para a implementação das DC possibilita que eles se sintam parte do processo (SCOTT et al, 2004; BAKER; FEDER, 1997).

Considerando ainda as evidências disponíveis, Grinshaw et al (2004) verificam que é necessário que os serviços identifiquem qual o melhor conjunto de estratégias a adotar, organizando um processo bem planejado e executado, uma vez que seu custo é significativo ao depender da disponibilidade de tempo dos profissionais e do adequado provimento de materiais e infraestrutura. As falhas na implementação de DC podem ser decorrentes tanto do planejamento quanto de sua execução (FIXSEN et al., 2005).

Os gestores entrevistados compartilharam da compreensão de que diversas estratégias devem ser buscadas e os esforços relatados parecem ser fruto da experiência de cada gestor e da cultura do serviço. A consistência dos processos parece ainda pouco estruturada. Houve um posicionamento muito favorável dos gestores à discussão como atividade para implementar as DC, sendo que grande parte deles avaliou que os profissionais aderem de forma mais intensa às atividades de discussão do que às aulas teóricas.

Ceccim e Feuerwerker (2004), fazendo uso do conceito de aprendizagem significativa, lembram que os processos de educação devem promover e produzir sentidos; que devem se basear na reflexão crítica, na problematização dos processos de trabalho reais, envolvendo os trabalhadores e os gestores. Tal processo não se esgota, gerando um contínuo de reflexão, aprendizado e intervenção nos serviços em todos os aspectos necessários para gerar qualidade desses serviços e valor para a população. Esse tipo de concepção não parece estar presente no discurso dos entrevistados; no entanto, a valorização da participação dos profissionais pode ser considerada um ponto favorável a ser explorado, ampliando a compreensão de como implementar mudanças nesses serviços.

A distribuição de material escrito tem sido considerada na literatura como indutor fraco de mudança das práticas dos profissionais, mas que deve ser utilizado, pois se enquadraria como um primeiro momento da disseminação das DC (GRIMSHAW et al., 2004; BERO et al., 1988; VILLAR; CARROLI; GULMEZOGLU, 2001). A qualidade do conteúdo e da forma é relevante para 
a receptividade do material pelos profissionais, o que reforça a necessidade de se proverem recursos adequados à produção de materiais nos serviços.

A escolha distinta dos gestores das redes em relação ao uso da internet decorre principalmente da infraestrutura dos serviços, uma vez que sua aceitação e uso foram relatados por gestores de ambas as redes, inclusive com acesso em seus domicílios para produzir material. A informatização foi também uma recomendação de todos os gestores para a melhoria dos serviços de saúde.

A análise do desempenho do serviço através da revisão e discussão de casos e óbitos foi descrita como tendo um efeito modesto (GRIMSHAW et al., 2004). Trata-se de uma sistemática que tem sido valorizada na literatura de qualidade, fazendo parte de um ciclo de planejamento e gestão, respondendo à necessidade de compreender os resultados para confirmar ou alterar os processos de trabalho, sendo utilizada nos esforços de uma expressiva rede de UTIN (HORBAR; PLESK; LEAHY, 2003), ampliando a possibilidade de gerar discussóes significativas sobre o cuidado, possibilitando a identificação de potenciais boas práticas e a melhoria dos resultados do cuidado oferecido.

Para Grinshaw et al. (2004), a utilização de lembretes foi descrita como de efeito moderado e valorizada como uma das estratégias que deve ser considerada. $\mathrm{O}$ desenvolvimento tecnológico na área de informática também estimula e possibilita o uso de diversas versões dessa técnica (GALLAGHER, 2002). As opções informatizadas para os lembretes não estavam acessíveis às unidades; as versões escritas (bilhetes e cartazes) não foram valorizadas pelos gestores, provavelmente devido aos meios precários utilizados. A racionalidade que embasa o uso dos lembretes é o apoio aos profissionais durante o cuidado, lembrando-os das DC. No entanto, o contexto das unidades pesquisadas não favorece seu uso. A superação dessa contradição poderia contribuir para a melhoria da qualidade do cuidado.

A interação com um profissional reconhecido - um expert - tem sido descrita como uma estratégia importante para o convencimento da equipe (GRIMSHAW et al., 2004) e utilizada pela RBPN, aproveitando oportunidades ao conciliar a vinda de um profissional de outros centros para diversas atividades acadêmicas, superando, dessa forma, em parte, a dificuldade de seu financiamento. A formalização no sistema de saúde desse tipo de estratégia seria factível em relação ao aprimoramento das redes locais, regionais, em que centros de referência 
poderiam manter contato mais próximo com os serviços de sua região, apoiando qualificação da assistência e a referência entre os serviços.

A utilização de diversas estratégias para melhorar o controle de infecção em uma UTIN foi utilizada por Bueno (2008), que durante seis meses desenvolveu reuniōes nas quais os profissionais podiam discutir conceitos e informações sobre as taxas de infecção da UTIN. Foram também fornecidos materiais educacionais como cartazes e folhetos. O autor conseguiu identificar melhora em alguns indicadores de infecção e de cuidado neonatal - embora não se tenha obtido redução da mortalidade -, reafirmando a importância da educação continuada dos profissionais, caracterizada pela utilização de diversas abordagens.

O tempo de que os gestores e os profissionais dispõem para as atividades como reuniões, revisão de casos e manutenção de sistema informatizado, tornase a questão mais relevante para os entrevistados, provavelmente motivando sugestôes de que o tempo para discussão seja incluído no cálculo da carga horária de trabalho e de que a lotação dos profissionais deveria ser tal que possibilitasse esse período fora da assistência direta ao paciente. Essa concepção - de lotação de profissionais - tem impacto econômico para os serviços e para o sistema de saúde; no entanto, a operacionalização destas estratégias deveria considerar esse aspecto prático, posição relatada também por Caminiti et al. (2005).

\section{Conclusão}

Os gestores entrevistados tendem a concordar com posicionamentos encontrados na literatura, tais como a utilização de diversas estratégias de implementação de DC e a ênfase na discussão em equipe. Contudo, parecem priorizar tais atividades a partir de suas reflexôes e experiência gerencial, não contando com estrutura favorável, apoios especializados e financiamento específico que ampliassem o potencial dessas ações.

Caberia incluir, no financiamento do sistema, a melhoria das condiçôes estruturais, especialmente a previsão de horário para a participação dos profissionais nas discussões nos serviços e também o suporte para o desenvolvimento das estratégias de implementação das DC, utilizando dessa forma mais uma ferramenta na busca da redução da distância entre o que existe de conhecimento e o que está disponível aos brasileiros ${ }^{1}$. 
ATALLAH, A.N. Medicina baseada em evidências: o elo entre a boa ciência e a boa prática clínica. In: - Caminhos do pensamento: epistemologia e método. Rio de Janeiro: Fiocruz, 2002, p.325 a 344 (Coleção Criança, Mulher e Saúde).

BAKER, R.; FEDER, G. Clinical guideline: Where Next? Int J Qual Health Care, n.9, p.399-404, 1997.

BERO, L.A. et al. Closing the gap between research and practice: an overview of systematic reviews of interventions to promote the implementation of research findings. BMJ, v.317, n.7156, p.465-74, 1988.

BUENO, A.C. Implantação de potenciais boas práticas de prevenção de infecção nosocomial em uma neonatal do município do Rio de Janeiro. Tese (Doutorado) - Instituto Fernandes Figueira, Fiocruz, Rio de Janeiro, 2008.

CABANA, M.C. et al. Why don't physicians follow clinical practice guidelines? A framework for improvement. JAMA, v.282, n.15, p.1458-65, 1999.

CAMITINI, C. et al. How to promote, improve and test adherence to scientific evidence in clinical practice. BMC Health Services Research, v.5, n.62, 2005.

CECCIM, R.B.; FEUERWERKER, L. O quadrilátero da formação para a área de saúde: ensino, gestão, atenção e controle social. Physis: Revista de Saúde Coletiva, v.14, n.1, jan-jun, 2004.

COMMITEE ON FETUS AND NEWBORN. American Academy of Pediatrics. Pediatrics v.114, p.1341-47, 2004.

DIJKSTRA, R. et al. The relationship between organizational characteristics and the effects of clinical guidelines on medical performance in hospitals, a meta-analysis. $B M C$ Health Services Research, v.6, n.53, 2006.

FIXSEN, D.L. et al. Implementation Research: a synthesis of the literature. Tampa: University of South Florida, 2005 (FMHI Publication \# 231).

GALLAGHER, E.J. How well do clinical practice guidelines guide clinical practice? Ann Emerg Med, v.40, n.4, p.394-98, 2002.

GRECO, P.J.; EISENBERG, J.M. Changing physicians' practices. $N$ Engl J Med., v.329, n.17, p. 1271-3, 1993.

GRIMSHAW, J. et al. Effectiveness and efficience of guideline dissemination and implementation strategies. Health Technol Assess, v.8, n.6, 2004.

HORBAR, J.D.; PLESK, P.E.; LEAHY, K. Establishing habits for improvement in neonatal intensive care. Pediatrics, v.111, n.4, p.e397-e410, 2003. 
MEDEIROS, M.L.B. Conhecimentos e práticas da displasia broncopulmonar em unidade neonatal pública do município do RJ. Dissertação (Mestrado) - Instituto Fernandes Figueira, Fiocruz, Rio de Janeiro, 2006.

MOTTA, P.R. Gestão contemporânea: a ciência e a arte de ser dirigente. 16a Ed. Rio de Janeiro: Record, 1991.

BRASIL. Ministério da Saúde. Portaria MS/GM 332, de 24 de março de 2000, retificação da portaria GM/MS 3.432, de 12 de agosto de 1998. Trata de critérios para UTI Neonatais. PRESTES, A.C.Y. et al. Frequência do emprego de analgésicos em unidades de terapia intensiva neonatal universitárias. J Pediatr, Rio de Janeiro, v.81, n.5, p.405-10, 2005.

REDE BRASILEIRA DE PESQUISAS NEONATAIS. Uso de corticosteróides e condições de nascimentos de pré-termos nos hospitais da Rede Brasileira de Pesquisas Neonatais. $R B G O$, v.26, n.3, p.177-84, 2004.

SCOTT, I.A. et al. Improving clinical practice: toward more effective use of decision support in clinical practice: what guidelines for guidelines don't tell you. Internal Medicine Journal, v.34, n.8, p.492-500, 2004.

TAMBLYN, R.; BATTISTA, R. Changing clinical practice: which interventions work? J Contin Educ Health Prof, v.13, n.4, p.273-88, 1993.

VILLAR, J.; CARROLI, G.; GULMEZOGLU, A.M. The gap between evidence and practice in maternal healthcare. Int Journal of Gynecology \& Obstetrics, v.75, supl 1, p.S47-S54, 2001.

WALLACE, L.S. et al. Development and Content Validation of Family Practice Residency Recruitment Questionnaires. Fam Med,v.35, n.7, p.496-8, 2003.

WOOLF, S.H. et al. Potencial benefits, limitation and harms of clinical guidelines. $B M J$, v.3, n.7.182, p.527-30, 1999.

\section{Nota}

${ }^{1}$ M.A.S.M. Gomes, S.M. Wuillanume e C. Magluta contribuíram igualmente com a concepção, análise dos dados, redação e revisão do texto. 


\section{Abstract}

Knowledge and practice in Brazilian neonatal ICU: the perspective of their managers on the implementation of clinical guidelines

Overcoming the gap between the established and systematized knowledge in clinical guidelines and practice of health services is a challenge to the quality of care. Clinical guidelines are tools that systematize the scientific evidence, helping to identify those relevant to the proper care. Several strategies to expand the use of clinical guidelines were identified in the literature: distribution of educational materials; meetings for training; review of practices; visits of experts; and reminders. The analysis of such strategies from the perspective of NICU managers was the object of study, developed in public NICU's through the application of semi-structured questionnaire with 53 managers (medical and nursing). Respondents reported the concomitant use of various activities / strategies, agreeing with placements in the literature. About $40 \%$ of heads felt that the distribution of written material encourages professional adhesion. Most managers reported that often uses the meetings to discuss clinical guidelines, which is a recommended activity for more than $95 \%$ of them. In the context studied, it was found that strategies to expand the dissemination of knowledge depend on the professionals themselves and services, with no formal institutional support for their financing, which reduces the consistency of its use.

Key words: Clinical Practice Guidelines; implementation; neonatology. 